\title{
LA FORMACIÓN EN MATERIAS DE INTELIGENCIA EN ESPAÑA: ESTADO DEL ARTE A FINALES DE 2015
}

\author{
Gustavo Diaz Matey y María Barco ${ }^{1}$ \\ UNISCI
}

\begin{abstract}
Resumen:
Todo lo relacionado con la inteligencia ha ido incorporándose progresivamente al debate público de las sociedades democráticas. España no es un caso aparte. En los últimos años, la oferta formativa relacionada con los temas de inteligencia, desde las más variadas perspectivas y desde distintas disciplinas, ha aumentado de forma exponencial. En este estudio hemos analizado la oferta formativa en España del 2012 al 2015 y llegado a diferentes conclusiones que representan el estado de los estudios de inteligencia en la actualidad y el camino a recorrer
\end{abstract}

Palabras clave: Estudios de Inteligencia, cultura de Inteligencia, entrenamiento, formación.

Título en Inglés: "The Intelligence Studies in Spain: The state of the art at the end of 2015"

\begin{abstract}
:
Everything related to intelligence has been progressively getting into the public debate on democratic societies. Spain is not a separate case. In the last few years the courses on intelligence offered, with different perspectives and disciplines have increased exponentially. In this study we analyze the courses offered on different intelligence topics in Spain from 2012 to 2015, reaching several conclusions on the state of the art on intelligence studies and the road ahead
\end{abstract}

Keywords: Intelligence Studies, culture of intelligence, training, teaching

Copyright (C) UNISCI, 2016.

Las opiniones expresadas en estos artículos son propias de sus autores, y no reflejan necesariamente la opinión de UNISCI. The views expressed in these articles are those of the authors, and do not necessarily reflect the views of UNISCI.

\footnotetext{
${ }^{1}$ Gustavo Díaz Matey es professor de Relaciones Internacionales en la Facultad de CC. Política y Sociología de la Universidad Complutense. María Barco posee el Grado en Relaciones Internacionales

E-mail: gdiazmat@ucm.es

DOI: http://dx.doi.org/10.5209/rev_RUNI.2016.n41.52674.
} 


\section{Introducción ${ }^{2}$}

Es más que evidente que los estudios de inteligencia han venido experimentando una auténtica revolución en las últimas décadas. Es más, desde finales de los años ochenta del siglo XX, y a pesar de que el secreto sigue jugando un papel muy relevante en todo lo relacionado con la inteligencia, ésta ha ido incorporándose progresivamente al debate público de las sociedades democráticas. Lo que en última instancia se ha visto reflejado con el desarrollo de distintos cursos y actividades en distintas universidades y organizaciones. ${ }^{3}$

España no es un caso aparte. En los últimos años, la oferta formativa relacionada con los temas de inteligencia, desde las más variadas perspectivas y desde distintas disciplinas, ha aumentado de forma exponencial. En este sentido, como ya hemos planteado en otros estudios, en los últimos años hemos asistido a un gran desarrollo de los estudios de inteligencia en España.

Así, en los últimos 25 años en los medios de comunicación y sectores académicos, gracias a la labor de los Servicios de Inteligencia españoles, del propio Ministerio de Defensa y del compromiso de distintos miembros de la comunidad académica española, se ha conseguido tratar el tema con discusiones, escritos, el desarrollo de diversas tesis doctorales, la creación de una Cátedra y un Instituto universitario sobre estas materias. ${ }^{4} \mathrm{~A}$ todo ello hay que sumar distintas iniciativas privadas, que han ido completando el actual Estado del Arte de los Estudios de inteligencia en España.

En este sentido, con el fin de avanzar en el desarrollo teórico de los estudios de inteligencia consideramos necesario describir y analizar la oferta de los estudios de inteligencia en España en relación a su calidad y a su relación con la demanda (carrera profesional). Esto nos permitirá desarrollar la idea de la necesidad de la creación de unos estándares mínimos para desarrollar este tipo de cursos, pero también ayudar a que la demanda entienda para qué puede servir estudiar "inteligencia". En ambos casos, algo a tener en cuenta, será entender cómo dar el paso de campo de estudio a disciplina científica y aunque ese camino puede que nunca se recorra debemos saber cuáles son sus veredas y a dónde nos conduce.

Para ello, hemos tomado como referencia la oferta formativa de los años 2012 a 2015 realizando una búsqueda exhaustiva (el proceso de búsqueda de información en relación a los cursos terminó a finales de 2015, tomando como referencia los cursos ofertados en esa fecha para el curso 2014/2015) de toda aquella oferta formativa vinculada a este campo, con independencia de su formato, duración o temática particular, así como de la naturaleza de la entidad organizadora o método de impartición. Esta búsqueda se ha visto completada, en algunos casos, con la información facilitada por los distintos directores y organizadores de los

\footnotetext{
${ }^{2}$ Para el desarrollo de este trabajo hemos compendiado los distintos cursos ofertados en relación a materias de inteligencia en España desde los años 2012 hasta el finales de 2015. Esta descripción ha tratado de ser lo más inclusiva posible y recoger todos los cursos ofertados. Si por cualquier razón se detectase un curso que se ha pasado por alto, no dude en ponerse en contacto con nosotros gdiazmat@,ucm.es

${ }^{3}$ Díaz Matey, Gustavo: “The Development of the Intelligence Studies in Spain”, International Journal of Intelligence and Counterintelligence, Vol. 23, $\mathrm{n}^{\circ}$ 4, (2010), p.749

${ }^{4}$ Blanco Navarro, José María; Díaz Matey, Gustavo: "Presente y Futuro de los Estudios de inteligencia en España”, Documento Marco 11/2015, IEEE, (2015), p.11, en:

http://www.ieee.es/Galerias/fichero/docs marco/2015/DIEEEM11-2015 EstudiosSeguridadEspana JMBlancoGustavoDiaz.pdf
} 
cursos a los autores. De este modo, el anexo anejo al final de este documento trata de aportar al lector una panorámica lo más amplia posible de los estudios de inteligencia en nuestro país.

Pese a la vocación inclusiva del presente estudio, ha quedado excluida aquella oferta formativa que, haciendo referencia explícita o implícita al concepto inteligencia, no brinda en sus programas atención alguna al estudio de la inteligencia o a la adquisición de habilidades vinculadas a su desempeño profesional. Su incorporación no podría más que generar ruido e incluir datos tanto cuantitativos como cualitativos no pertinentes, distorsionando los objetivos anteriormente señalados. Se ha optado por introducir únicamente aquella formación ofertada por entidades radicadas en territorio español, pese a la posibilidad para el público castellanoparlante de cursar diferentes cursos procedentes de la oferta formativa latinoamericana impartida online, por ejemplo.

A la hora de llevar a cabo la evaluación de los estudios de inteligencia en España debemos tener en cuenta en primer lugar, cuáles son los criterios que se aplican tanto a nivel europeo como nacional para medir la calidad de las enseñanzas superiores en nuestro país. De mismo modo, es relevante el análisis comparativo de las enseñanzas de inteligencia con otros países de nuestro entorno, en los que este tipo de estudios están mucho más desarrollados.

Como paso previo al análisis debemos acudir a los criterios desarrollados por el Espacio Europeo de Educación Superior (ESG), ${ }^{5}$ cuyos parámetros son los empleados por las distintas agencias de evaluación adscritas a nivel nacional. Estos estándares se dividen en tres grupos distintos: aquellos relativos a la garantía de la calidad interna de las enseñanzas; los que se refieren a la garantía de la calidad externa, y por último, lo estándares que se aplican a las agencias de garantía de la calidad. En el caso que nos ocupa, únicamente nos interesan aquellas variables que hacen referencia a la calidad interna de las enseñanzas.

En el caso de España, aunque existen distintas agencias de calidad (la Agencia Nacional de la Evaluación de la Calidad más las Agencias Autonómicas $)^{6}$ que se acogen a los parámetros planteados por la ESG, tomaremos como referencia a la Agencia Nacional de Evaluación de la Calidad y Acreditación (ANECA), al ser el organismo que se encuentra más extendido. Los parámetros empleados por la ANECA son similares a los planteados en los documentos de la ESG, aunque descritos de forma exhaustiva.

Este organismo señala diez criterios y directrices para medir la calidad de las enseñanzas superiores: descripción del título; justificación; competencias; acceso y admisión; planificación de las enseñanzas; personal académico; recursos materiales y servicios; resultados previstos; sistemas de garantía de calidad y por último, calendario de implantación. Estos criterios hacen referencia a aspectos estructurales y formales del título.

\footnotetext{
${ }^{5}$ Informe de ENQA sobre Criterios y Directrices para la Garantía de Calidad en el Espacio Europeo de Educación Superior, European Association for Quality Assurance in Higher Education, 2005, Helsinki.

${ }^{6}$ Cataluña - Agència per a la Qualitat del Sistema Universitari (AQU); Islas Baleares - Agència de Qualitat Universitària (AQUIB); Canarias - Agencia Canaria de Evaluación de la Calidad y Acreditación Universitaria (ACECAU); Andalucía - Agencia Andaluza de Evaluación de la Calidad y Acreditación; Andalucía - Unidad para la Calidad de las Universidades Andaluzas (UCUA); Castilla y León - Agencia para la Calidad del Sistema Educativo Universitario; Galicia - Axencia para a Calida de do Sistema Universitario de Galicia (ACSUG); Comunidad Valenciana - Comisión Valenciana de la Acreditación y Evaluación de la Calidad; País Vasco - Agencia de Evaluación y Acreditación de la Calidad del Sistema Universitario; Aragón - Agencia de Calidad y Prospectiva Universitaria de Aragón
} 
Por otro lado, además de los organismos evaluadores de carácter público se han tenido en cuenta las aportaciones de otro tipo de organizaciones cuyo objetivo principal es el intercambio y profundización de conocimientos sobre las enseñanzas de inteligencia. España no cuenta con ninguna asociación de este tipo que presente de forma pública criterios similares, ${ }^{7}$, por lo que hemos tomado como referencia la International Association for Intelligence Education (IAFIE). ${ }^{8}$ Esta organización norteamericana propone una serie de estándares que deben estar presentes en cualquier estudio de inteligencia, tanto de grado como en títulos superiores. ${ }^{9}$ Los estándares desarrollados por esta organización se centran sobre todo en los aspectos teóricos y de contenido que deben recoger los estudios superiores de inteligencia.

Dado que ninguno de los cursos sobre inteligencia que se ofertan en España ha conseguido la certificación de Máster Oficial, (que permita el acceso a estudios de doctorado) ha sido necesario adaptar los criterios que iban a emplearse para su análisis. Nos hemos centrado en cuatro variables para analizar la calidad de dichos estudios en España que guardan relación con los criterios planteados por la ANECA, es decir, los aspectos más formales. No obstante, aquellos apartados donde se hace referencia al contenido del título hemos tenido en cuenta los estándares desarrollados por la IAFIE, además de dar una visión comparada de los estudios de inteligencia con países donde esta formación se encuentra mucho más arraigada.

El primero de los criterios identificados hace referencia a la justificación del título, la descripción que se da del mismo, y si los objetivos principales señalados en las competencias quedan reflejados en el programa del título. Con respecto a este criterio el objetivo principal ha sido observar si el título del curso guarda coherencia con el contenido del mismo. En segundo lugar, hemos procedido al análisis de los programas de estudio y sus asignaturas, con el fin de destacar las posibles carencias que presentan los mismos, si estos guardan relación con las competencias previamente establecidas, y si cuentan con una estructura interna relativa a la planificación temporal de los estudios. En tercer lugar, el profesorado encargado de impartir estos cursos de posgrado. Por último, nos hemos centrado en observar las fechas de implantación y duración de los estudios.

A la hora de recabar la información sobre las variables señaladas anteriormente, es necesario señalar como denominador común a todas ellas la escasa información encontrada en las distintas páginas web de información de los estudios ${ }^{10}$.

Se han identificado 67 acciones formativas públicas, categorizadas en jornadas, talleres, cursos (modulares, de especialización y de postgrado) y masters (títulos propios). Entre estos últimos, cabe destacar que ninguno de ellos tiene carácter de Máster Oficial reconocido por la ANECA $^{11}$, puesto que no cumple con los criterios planteados por esta institución. Por

\footnotetext{
${ }^{7}$ Un ejemplo aparece en: Asociación Analistas de Inteligencia, en: http://www.analistasinteligencia.es/; Asociación Española para la promoción de la inteligencia competitiva, en: http://www.asepic.com.es/

${ }^{8}$ Asociada a esta organización encontramos la IAFIE-EUROPE, (donde España no cuenta con participación activa), fundada en julio de 2015, con objetivos similares aunque su labor se encuentra mucho menos desarrollada debido a su reciente creación.

${ }^{9}$ International Association for Intelligence Education, Standards for Intelligence Education Undergraduate and Graduate Programs, en: http://www.iafie.org/?page=IntelEd

${ }^{10} \mathrm{Al}$ final del documento se adjunta la relación de cursos ofertada en España con los enlaces para poder acceder a toda la información.

${ }^{11}$ Real Decreto 1393/2007, de 29 de octubre, por el que se establece la ordenación de las enseñanzas universitarias oficiales, en: https://www.boe.es/buscar/act.php?id=BOE-A-2007-18770
} 
consiguiente, estos títulos no dan acceso a los estudios eurses de doctorado en una universidad española, por lo que a pesar de ser denominados masters deberíamos hablar, de la figura de Máster Propio, aunque en el trabajo hemos englobado toda esta formación de postgrado, por razones de simplicidad, en títulos propios de posgrado independientemente de su duración o formato. En general, los horarios y fechas están adaptados a gente que compagine estos estudios con el horario laboral, y su precio medio ronda los $100 €$ por crédito ECTS.

En relación con las organizaciones que constituyen la oferta formativa en materias de inteligencia en España se han encontrado veintiocho entidades de diversa naturaleza: once universidades, ocho empresas privadas, tres escuelas de negocio, dos institutos universitarios, una fundación, una asociación, un centro de estudios ligado al Ministerio de Defensa, y hasta una entidad bancaria.

\section{2.- Características de la oferta formativa en España.}

Tras el análisis de los distintos cursos por medio de las variables nombradas anteriormente, podemos señalar los rasgos principales de los títulos sobre inteligencia ofertados en España:

Se evidencian varias tendencias resaltables en el tipo de oferta y evolución de la misma. En general, se destaca un mayor interés empresarial por los estudios de inteligencia en los últimos años. Sin embargo, esto no se ha traducido en un aumento relevante de la demanda. La poca estabilidad que muestra la oferta formativa, como explicaremos a continuación, hace que la demanda no sea tan llamativa como podría esperarse, ya que han sido pocas las entidades capaces de capitalizar dicha tendencia. Lejos de poder reducirse únicamente a una explicación cuantitativa de armonización entre oferta y demanda (número de plazas ofertadas y número de interesados solventes en realizar cualquiera de los cursos) se debe a razones fundamentalmente cualitativas, entre las que destacan:

- El alto grado de inestabilidad de la oferta. La variabilidad que se hA experimentado en el transcurso de los años ha llevado a que cerca de la mitad de los cursos abiertos a matrícula en el año 2012 - veintiocho de ellos - hayan desaparecido para el presente curso académico. De los cursos que no han tenido continuidad, catorce de ellos se impartían a través de medios online, doce adoptaban una metodología presencial, uno semipresencial y otro ofrecía la opción a elegir entre diferentes modalidades. Mientras muchos de ellos han desaparecido, otros han cambiado su materia y su denominación. También hay que mencionar aquellos cursos que ni quiera vieron la luz, como una iniciativa que no llego a ver la luz desarrollada por el Instituto Empresa en el Curso 2013/2014.

- La dispersión temática existente. Buena prueba de la falta de madurez que afecta al sector. A grandes rasgos, que de forma generalizada el sector es incapaz de ofrecer una formación de rigor y con proyección suficiente como para habilitar profesionalmente al alumnado. Este hecho solo puede conducir a la frustración de las expectativas de los estudiantes (que a menudo han sido alimentadas interesadamente) haciendo aumentar su descontento ${ }^{12}$. Estos deciden cursar este tipo de formación, desembolsando en ocasiones una cantidad importante de dinero con la intención de mejorar sus futuras condiciones de empleabilidad. Esta tendencia afecta especialmente a los títulos propios que bajo el epíteto de "Master" son lanzados por diferentes universidades españolas, tanto públicas como privadas.

12 A este respecto ver: Comunicado. España necesita 1.000 especialistas en inteligencia, en: http://www.europapress.es/comunicados/empresas/noticia-comunicado-espana-necesita-1000-especialistasinteligencia-20141006141056.html 
- El recelo al término de inteligencia por parte de algunas universidades españolas. La falta de claridad conceptual de lo que se entiende por inteligencia lleva a algunas entidades a no apostar por incluir en su oferta formativa cursos vinculados a esta temática.

Existen distintas razones para afirmar la falta de interés de algunas entidades en formación relacionada con cuestiones de inteligencia. Dos cuestiones derivadas de la falta de una verdadera cultura de inteligencia en nuestro país. En primer lugar, la propia percepción de lo que se tiende a entender por inteligencia, relacionándola frecuentemente con teorías conspirativas y fantasías de espías, hace que distintos organismos vean este tipo de cursos como "poco serios" para el currículo de sus instituciones. En segundo lugar, la percepción errónea, a nuestro juicio, de la utilidad real y las salidas profesionales de la inteligencia como desempeño profesional, lo que unido al carácter eminentemente teórico de los cursos hace que no solo el alumnado, sino también las propias entidades ofertantes no vean una necesidad real de ofertar este tipo de itinerarios formativos.

- Por último, debido a la dificultad que encuentran los propios investigadores en el campo de la inteligencia (ante la falta de oportunidades en las propias universidades o ante el limitado acceso a fuentes primarias en este campo, derivado de la ausencia de una política de desclasificación en España), existe una carencia real de profesorado capacitado en este campo de estudio en la mayoría de las instituciones. Ello dificulta el desarrollo de cursos con profesores ajenos a la universidad que en muchas ocasiones poco o nada tienen que ver con la academia y que cuentan con poca o ninguna experiencia docente. Y como todos sabemos, no es lo mismo hacer que enseñar a hacer.

Si en el año académico 2012/2013 se ofertaban un total de seis cursos de este tipo de titulaciones, en el 2014/2015 el total de cursos ofertados es de diez. En este último año académico, 2014-2015, es cuando se ha producido un aumento más agudo de este tipo de oferta formativa, con la apertura de masters como el Máster en Inteligencia Económica de la Universidad Autónoma de Madrid, el Máster en Inteligencia Global y Nuevas Tecnologías de la Universidad de Barcelona, o el Máster Internacional en Ciberseguridad, Ciberdefensa e Inteligencia en Redes Digitales de la Universidad a Distancia de Madrid en colaboración con el Campus Internacional para la Seguridad y la Defensa. Es esta última entidad, junto a la Universitat Abat Oliva CEU y la Fundación de Estudios Estratégicos e Internacionales, la que se ha convertido en el curso académico 2014/2015, en el principal centro ofertante de títulos propios de posgrado bajo denominación de Máster (dos masters), como de cursos de carácter especializado (trece cursos), todos ellos impartidos a distancia.

A la oferta reciente se suma el "Máster Interuniversitario en Analista de Inteligencia" ofertado por cuatro universidades españolas bajo el sello del Centro Nacional de Inteligencia y que se encuentra hoy en su VII Edición, siendo esta la formación dedicada a los estudios de inteligencia más longeva y asentada de nuestro país.

Por su parte, la Universidad Autónoma de Madrid (UAM) ha ofertado en 2015 un curso de experto en análisis de inteligencia, que se encuentra hoy en su segunda edición, habiéndose ofertado anteriormente como ciclo de formación. Además, la UAM ofrece la posibilidad de realizar de forma independiente cada uno de los módulos que componen sus dos cursos, pasando a ser la universidad con mayor oferta formativa sobre diferentes aspectos vinculados a los estudios de inteligencia (doce cursos).

Para poder realizar un breve análisis comparativo de los estudios de inteligencia a nivel internacional se ha tomado como ejemplo Reino Unido, siendo uno de los países donde los estudios sobre inteligencia se encuentran más arraigados. Encontramos un elevado número 
de universidades a la largo de todo el país que ofertan Masters sobre inteligencia. ${ }^{13}$ En las distintas páginas web donde se ofertan estos estudios encontramos información exhaustiva sobre los mismos, ya sea en lo referido a aspectos formales como a plazos de admisión o al comienzo de los distintos cursos, como en el plano conceptual, con una descripción detallada de los distintos módulos de los que se compone. Al compararlo con las entidades españolas que promocionan estos cursos, la información disponible es mucho mayor y se encuentra de forma clara y sencilla.

\section{3.- El papel de la formación online}

En el curso académico 2012-2013 podían encontrarse un total de sesenta cursos, de los cuales la mitad se impartían a distancia a través de medios online. Veinticuatro adoptaban una metodología presencial, tres semipresencial, y tres ofrecían la opción de realizar el curso en diferentes modalidades.

El ratio entre cursos online y presenciales sigue manteniéndose en el tiempo, siendo los cursos online la prioridad en la oferta. Sin embargo, una observación detenida, muestra cierta superficialidad en la elaboración de los cursos online. Esta superficialidad de los cursos online es consecuencia, principalmente, de la propia naturaleza de este tipo de enseñanza, donde debido a la corta duración de los mismos, queda en evidencia que este tipo de cursos no cuenten con un contenido sólido y original sin una conexión necesaria en otros campos de las Ciencias Sociales. Así, proliferan los pequeños cursos sobre temáticas particulares, sin estar encuadrados en un itinerario formativo mayor o, lo que es más grave, sin atender con rigurosidad y exhaustividad a los axiomas más fundamentales del estudio de la inteligencia. Todo ello unido a la falta de contenido práctico de los mismos hace que el éxito de lo que ya existe sea muy cuestionable y que la consolidación de los mismos en el tiempo, salvo excepciones y matices, sea muy limitada.

A la hora de ofertar cursos cortos, la mayoría son talleres presenciales. Pero a la hora de presentarlos online, los cursos cortos pertenecen únicamente a bloques de materias sacados de cursos más extensos y debido a ello quedan descontextualizaos y en ocasiones vacíos. Por último, a pesar de que varios de estos cursos se ofertan con un carácter eminentemente práctico, existe una marcada ausencia de prácticas profesionales en todos ellos. En el caso de los cursos online esta ausencia es, si cabe, aún más destacada.

Por otro lado, el desarrollo de la oferta online ha abierto un abanico de cursos temáticos especializados, ofertados tanto por universidades como por escuelas de negocios o empresas. Sin embargo, en escasas ocasiones estos cursos abordan los aspectos troncales de los estudios de inteligencia, por lo que difícilmente pueden responder a las necesidades formativas de aquellos que ya cuentan con bases teóricas o experiencia.

\footnotetext{
${ }^{13}$ Ejemplo de los cursos de posgrado ofertados en Reino Unido para el curso 2016/2017: King's College London (MA Intelligence and International Security), en http:/www.kcl.ac.uk/study/postgraduate/taughtcourses/intelligence-and-international-security-ma.aspx ; Buckingham University (Security and International Studies), en http:/www.buckingham.ac.uk/humanities/ma/securityandintelligence ; University of Saldfor (MA Intelligence and International Studies), en http://www.salford.ac.uk/pgt-courses/intelligence-and-security-studies ; University of Glasgow (MA International Security, Intelligence and Strategic Studies), en http://www.gla.ac.uk/postgraduate/taught/internationalsecurity/ ; Brunel University London (MA Intelligence and Security Studies), en http://www.brunel.ac.uk/courses/postgraduate/intelligence-and-security-studies-ma.
} 
En el caso de Reino Unido la oferta de formación online es mucho más reducida. Sin embargo, en aquellas donde se permite la opción de realizar el curso a distancia, los contenidos que recogen son similares a los de los cursos presenciales.

\section{4.- Análisis de la temática de los cursos de inteligencia.}

En lo que respecta a la temática de cada curso, se constata cierta repetición de ideas y falta de rigor conceptual. Este argumento queda patente en el análisis de los contenidos de buena parte de la oferta formativa. Mientras se constata una sobredimensión del llamado ciclo de inteligencia no se abarcan las materias relacionadas con la aplicación real de este tipo de formaciones. Esto se debe principalmente a la falta del mencionado rigor conceptual, y a la debilidad teórica de un campo de estudio todavía emergente. De esta forma, mientras se destaca una excesiva fijación por técnicas y métodos especializados comprobamos que se abandona la profundización y el debate en la teoría, lo que en última instancia redunda en una falta de contextualización de los estudios de inteligencia en el marco general de las Ciencias Sociales.

Este punto es importante, ya que a la hora de estudiar cualquier tema en Ciencias Sociales el primer paso es establecer unas fronteras nítidas del objeto de estudio en cuestión. Estas sólo pueden establecerse de forma clara a través de la claridad conceptual, la cual otorga consistencia a su vez a métodos y procedimientos. Es a través de la claridad en los conceptos la forma que tenemos de llegar a definiciones suficientes que sustenten, en último término, relaciones entre distintos elementos. Son las definiciones las que ordenan nuestros conocimientos y nos ayudan a establecer un marco de referencia suficiente para posteriormente sacar conclusiones. Por tanto, todo campo de estudio que no aborde con suficiente rigurosidad la tarea de definir los conceptos que le son propios y establecer las relaciones existentes entre ellos difícilmente podrá alcanzar un grado de especialización y congruencia interna suficiente para superar los estrechos márgenes del conocimiento ordinario.

En esta línea, después de una profunda revisión de la oferta formativa existente y teniendo en cuenta que este no es un trabajo teórico, que no existe consenso alguno acerca de lo que se entiende por inteligencia, como evidencia la dispersión conceptual existente entre los contenidos de la oferta formativa. Ello dificulta enormemente establecer una frontera nítida de lo que incluirían los llamados estudios de inteligencia.

Se observa el predominio que tiene la obtención y análisis de la información en estos estudios, bajo la continua repetición de conceptos como fuentes, información, datos y, sobretodo, análisis. Por el contrario, no existe apenas formación en la diseminación y comunicación de la inteligencia. Es decir, falta capacitación en la interlocución con los clientes desde las primeras fases de identificación e interpretación de las necesidades de información, hasta las últimas fases relacionadas con la diseminación y feedback de los productos de inteligencia.

El uso repetido de términos militares (contrainteligencia, espionaje, terrorismo, etc.) denota un uso interesado como reclamo publicitario. Lo que en última instancia hace que se usen términos que nada tienen que ver con el campo de la inteligencia (sanitaria, israelí o turística). 
Se constata la reducción de términos muy extendidos habitualmente, como el de inteligencia competitiva. En concreto, la oferta formativa en relación a la inteligencia competitiva se ha reducido un $18 \%$ en comparación con los cursos del 2012/2013. Un término cada vez más en desuso entre otras razones por la desaparición de ofertas en España como las de Infocenter o Institute for Competitive Intelligence, y a pesar de que distintos masters (el de la URJC, el de la UAM o los del FESEI) tienen módulos sobre inteligencia competitiva como uno de sus reclamos. Desde distintos ámbitos institucionales como el ICEX, se aboga por diferenciar claramente el término inteligencia competitiva, inteligencia empresarial e inteligencia económica. Estos términos, aunque complementarios, no son en absoluto sinónimos.

En cuanto a la temática de los cursos ofertados por el Reino Unido, observamos una estructura bien diferenciada en los mismos entre módulos obligatorios que abordarían la parte teórica y conceptual relativa a los conocimientos de inteligencia; y en segundo lugar, una serie de módulos opcionales más específicos. Podemos observar una vez más como el acceso a la información relativa a los contenidos recogidos por los cursos es extenso.

\section{Conclusión.}

La oferta formativa en este campo en España ha dado pasos importantes en los últimos años, pero tras el estudio de los distintos contenidos en dicha oferta, podemos afirmar que falta coherencia y profundidad en los mismos. Hasta el momento el sector tiene una clara falta de madurez, con iniciativas formativas superficiales, con una falta de contextualización y del rigor necesario para poder trasmitir de forma contundente la utilidad de la inteligencia, con independencia de la estructura y del contexto temporal en el que se aplique.

Vemos en el contenido de los cursos, por ejemplo, una falta de continuidad argumental en los mismos de forma que quede claro qué implica estudiar inteligencia, qué materias básicas deberían tratarse (más allá del manido ciclo de inteligencia o de las técnicas estructuradas de análisis) y qué competencias se adquieren con estos cursos, necesarias para aplicarlas de forma profesional a según qué trabajos en según qué estructuras. El por qué puede encontrarse en la propia evolución de los estudios de inteligencia en España.

El desarrollo de la llamada cultura de inteligencia en España proviene del interés de los propios servicios de inteligencia por conseguir "adaptarse" a los nuevos tiempos y acercarse, de algún modo, a la ciudadanía dejando atrás la imagen de servicios de espionaje y acciones encubiertas. En este sentido, los servicios de inteligencia españoles ayudados por parte de la academia de este campo fueron conscientes, si bien tardíamente, de la progresiva revolución que han sufrido los estudios de inteligencia desde 1975 en adelante y lo que ha supuesto una mayor desclasificación y apertura para aquellos países que llevan a acabo tales procesos.

Así, a pesar de que tradicionalmente todo lo referente a estudios de inteligencia en España ha sido un tema tabú e incluso ha sido perseguido, sin que se hayan reparado notables abusos, existe un claro cambio de orientación a raíz del cambio de siglo. Poco a poco se ha ido fomentando una progresiva apertura controlada con dos objetivos principalmente. Dirigir la cultura de inteligencia y por tanto, establecer una línea oficial de los contenidos y los investigadores que deberían tratar en los distintos cursos de inteligencia y por otro lado, aplacar cualquier esfuerzo de desclasificación en nuestro país. 
Esto tiene consecuencias directas sobre el desarrollo de los estudios de inteligencia en nuestro país. En primer lugar, debido a esa falta de fuentes primarias y de líneas de investigación claras, existe una falta de consistencia en los contenidos que tratan los distintos cursos. Como ya hemos apuntado, los temas son tremendamente dispares, muchas veces contradictorios y cuanto menos poco profundos. Por este motivo, la desclasificación de archivos oficiales es una de las necesidades más acuciantes en la correcta expansión y avance de los estudios de inteligencia en España ya que constituye una forma única de acceder a fuentes primarias; sirviendo no sólo de indicador sobre el nivel de compromiso de Estado para con este campo de estudio; también como elemento clave para el desarrollo de unas bases teóricas claras y comunes y la contrastación de hipótesis. De hecho, a una mayor desclasificación, mayor desarrollo de los estudios de inteligencia y por ende una mayor conciencia de la cultura de inteligencia en el país que desclasifica. ${ }^{14}$

Por otro lado, llama la atención la falta de integración de los distintos estudios de inteligencia en España en los programas de estudio de las distintas facultades españolas. Salvo contadas excepciones los distintos programas y cursos no se integran en las distintas disciplinas científicas y por tanto, quedan como cuestiones aisladas sin un enganche claro en el resto de las Ciencias Sociales. Esto puede ser debido a la falta de estudiosos en el marco de la universidad española de este campo de estudio. Lo que en última instancia repercute en una falta de profundidad derivada de la falta de debate teórico y de claridad en las fronteras de lo que se entiende por inteligencia y sus ámbitos de actuación.

Sea como fuere estas reflexiones pueden ser más o menos acertadas y el lector puede estar más o menos de acuerdo con las mismas. Sin embargo, existe un problema fundamental que sobrevuela los estudios de inteligencia en España: la falta de salidas e integración de los distintos alumnos en el mercado laboral. Mientras lo que dice algún colega "España necesita 1000 especialistas en inteligencia" 15 no sea cierto, y no exista una política clara de desclasificación en este país los estudios de inteligencia seguirán siendo un campo de estudio subdesarrollado con grandes posibilidades de desaparecer o lo que es peor de convertirse en pasto de aproximaciones seudocientíficas denostadas sin ninguna base académica.

En este trabajo hemos descrito y analizado el Estado del Arte de los estudios de inteligencia en España. El futuro está por llegar, pero nos encontramos ante una oportunidad única de explorar un "nuevo" campo de carácter multidisciplinar y con innumerables aplicaciones en el sector público y privado. Es más, en un futuro próximo, si el propio desarrollo de los Estudios de Inteligencia es el correcto, veremos cómo este tipo de formación e iniciativas incidirán sustancialmente en el desempeño profesional de la inteligencia, lo que en última instancia repercutirá en el propio desarrollo de los estudios, retroalimentándose.

Sin embargo, hasta que estas iniciativas académicas y formativas no tengan un impacto en la profesión de inteligencia y contribuyan a su mejora de forma clara, este tipo de estudios quedarán relegados a un segundo plano y sus contenidos no se adecuarán a las necesidades básicas reclamadas por la propia profesión. Un paso clave para relacionar formación en inteligencia y desempeño profesional será conseguir consensuar unos estándares mínimos en

\footnotetext{
${ }^{14}$ McCartney, John. "Teaching Intelligence Getting started", American University and AFIO, (Marzo, 1999); Haines, Gerald K. “An emerging new field of study: US Intelligence”, Diplomatic history, Vol. $28, \mathrm{n}^{\circ} 3$ (June 2004), pp.441-449.

${ }^{15}$ Comunicado: España necesita 1.000 especialistas en inteligencia, en http://www.europapress.es/comunicados/empresas/noticia-comunicado-espana-necesita-1000-especialistasinteligencia-20141006141056.html
} 
la formación relacionada con inteligencia derivados de unos conceptos comunes y unas definiciones comunes. Algo que a día de hoy está muy lejos de suceder.

Esperemos que los estudios de inteligencia sean capaces de encontrar su propio camino en las Ciencias Sociales y terminen por convertirse en una disciplina propia aceptada por el resto de los investigadores científicos, e integrada en el resto de las disciplinas adyacentes. Para que los Estudios de inteligencia se puedan convertir en una disciplina aún falta mucho camino por recorrer es largo, pero es indudable que la escuela española de estudios de inteligencia está desarrollándose y en los próximos años veremos resultados. 


\section{Lista de la oferta formativa en España en 2015. Master Propio y Cursos de Posgrado}

\begin{tabular}{|c|c|c|c|c|}
\hline $\begin{array}{l}\text { Máster Interuniversitario en } \\
\text { Analista de Inteligencia. }\end{array}$ & $\mathbf{P}$ & $\begin{array}{c}\text { Universidad Rey Juan Carlos / } \\
\text { Universidad Carlos III / } \\
\text { Universitat Autónoma de } \\
\text { Barcelona / Universitat de } \\
\text { Barcelona }\end{array}$ & Sept- Dic & $\frac{\text { www.masteranalistadeintelige }}{\underline{\text { ncia.com }}}$ \\
\hline $\begin{array}{l}\text { Postgrado de Inteligencia } \\
\text { Económica y Seguridad. }\end{array}$ & $\mathrm{P}$ & $\begin{array}{c}\text { Universidad Pontificia / } \\
\text { Centro de Innovación del } \\
\text { Derecho }\end{array}$ & Nov & $\frac{\frac{\text { http://www.masconsulting.es/i }}{\text { nteligenciaeconomica/index.ht }}}{\underline{\mathrm{ml}}}$ \\
\hline $\begin{array}{c}\text { Máster en Inteligencia } \\
\text { Económica y Análisis Experto. }\end{array}$ & $\mathrm{P}+\mathrm{NP}$ & $\begin{array}{l}\text { Universidad Francisco de } \\
\text { Vitoria / Grupo ATENEA }\end{array}$ & Oct & $\begin{array}{l}\frac{\text { http://www.grupoateneasd.es/ }}{\text { wp- }} \\
\frac{\text { content/uploads/2014/02/INT }}{\text { ELG-ECON-MODULOS-19- }} \\
\text { FEB-2014-1.pdf }\end{array}$ \\
\hline $\begin{array}{l}\text { Máster en Liderazgo, } \\
\text { Gobernanza e Inteligencia } \\
\text { Económica y Empresarial. }\end{array}$ & $\mathrm{P}+\mathrm{NP}$ & $\begin{array}{c}\text { CEU Universitat Abat Oliba / } \\
\text { FESEI }\end{array}$ & Nov & $\frac{\text { http://www.fesei.org/files/pdfs }}{\text { /Master_Burgos.pdf }}$ \\
\hline $\begin{array}{l}\text { Máster en Inteligencia Global } \\
\text { y Nuevas Tecnologías. }\end{array}$ & $\mathrm{P}$ & $\begin{array}{l}\text { Universidad de Barcelona / } \\
\text { ENTI }\end{array}$ & Oct-Jul & $\frac{\underline{\text { htpp://mastereninteligencia.co }}}{\underline{\mathrm{m}}}$ \\
\hline $\begin{array}{l}\text { Máster en Inteligencia } \\
\text { Económica. }\end{array}$ & $\mathrm{P}$ & $\begin{array}{l}\text { Universidad Autónoma de } \\
\text { Madrid / ICFS }\end{array}$ & Oct - Dic & $\frac{\underline{\text { http://sei.icfs.es/master-de- }}}{\underline{\text { inteligencia-economica-0 }}}$ \\
\hline $\begin{array}{l}\text { Máster Internacional en } \\
\text { Inteligencia: gestión y análisis. }\end{array}$ & $\mathrm{P}+\mathrm{NP}$ & $\begin{array}{l}\text { Universidad Antonio de } \\
\text { Nebrija }\end{array}$ & Feb-Nov & $\begin{array}{l}\frac{\text { http://www.nebrija.com/progr }}{\text { amas-postgrado/titulos- }} \\
\frac{\text { propios/master-internacional- }}{\text { inteligencia/master- }} \\
\text { inteligencia.php }\end{array}$ \\
\hline $\begin{array}{l}\text { Máster en Operaciones de } \\
\text { Inteligencia y } \\
\text { Contrainteligencia. }\end{array}$ & NP & $\begin{array}{l}\text { Universidad a distancia de } \\
\text { Madrid / CISDE }\end{array}$ & Mar-Nov & 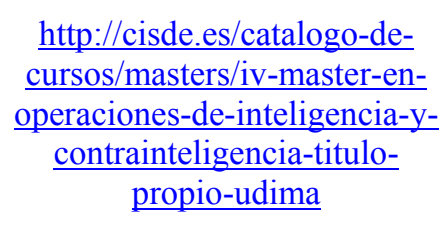 \\
\hline $\begin{array}{l}\text { Máster en Liderazgo, } \\
\text { Diplomacia e Inteligencia. }\end{array}$ & $\mathrm{P}+\mathrm{NP}$ & $\begin{array}{c}\text { CEU Universitat Abat Oliba - } \\
\text { FESEI }\end{array}$ & Nov & 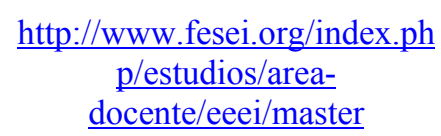 \\
\hline $\begin{array}{c}\text { Máster Internacional en } \\
\text { Ciber-seguridad, Ciber- } \\
\text { defensa }\end{array}$ & NP & $\begin{array}{l}\text { Universidad CEU San Pablo/ } \\
\text { CISDE }\end{array}$ & Sep & 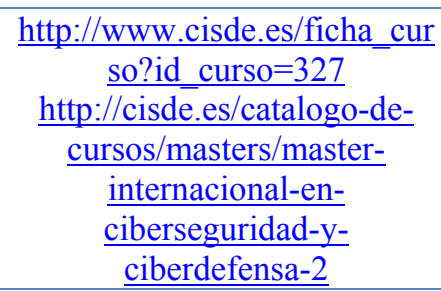 \\
\hline
\end{tabular}




\begin{tabular}{|c|c|c|c|c|}
\hline $\begin{array}{l}\text { Máster Universitario en } \\
\text { Seguridad, Defensa y } \\
\text { Geoestratégia }\end{array}$ & NP & UDIMA-CISDE & Oct-Jul & $\frac{\text { http://www.udima.es/es/maste }}{\frac{\text { r-seguridad-defensa- }}{\text { geoestrategia.html }}}$ \\
\hline $\begin{array}{l}\text { V Máster en Operaciones de } \\
\text { Inteligencia y } \\
\text { Contrainteligencia }\end{array}$ & NP & UDIMA-CISDE & Oct-Jul & $\begin{array}{l}\frac{\text { http://cisde.es/catalogo-de- }}{\text { cursos/masters/v-master-en- }} \\
\text { operaciones-de-inteligencia-y- } \\
\frac{\text { contrainteligencia-titulo- }}{\text { propio-udima }}\end{array}$ \\
\hline $\begin{array}{l}\text { Máster Propio en Estudios } \\
\text { Estratégicos y de Seguridad } \\
\text { Internacional }\end{array}$ & NP & Universidad de Granada & Nov-Sept & $\frac{\text { http://www.ugr.es/ jjordan/ma }}{\underline{\text { sterestudiosestrategicos.pdf }}}$ \\
\hline $\begin{array}{l}\text { Posgrado en Policía Científica } \\
\text { e Inteligencia Criminal }\end{array}$ & $\mathrm{P}$ & $\begin{array}{c}\text { Universidad Autónoma de } \\
\text { Barcelona. Escuela de } \\
\text { Prevención y Seguridad } \\
\text { Integral }\end{array}$ & Ene-Nov & $\begin{array}{l}\frac{\text { http://www.uab.cat/web/postg }}{\text { rado/diplomatura-de- }} \\
\begin{array}{c}\text { postgrado-en-policia- } \\
\text { cientifica-e-inteligencia- }\end{array} \\
\frac{\text { criminal/informacion-general- }}{1206597472083 . h t m 1 / \text { param1- }} \\
\underline{3002 \text { es } / \text { param2-2008/ }}\end{array}$ \\
\hline
\end{tabular}

\section{Otros Cursos}

\begin{tabular}{|c|c|c|c|c|}
\hline Denominación & Modalidad & Institución & Duración & Enlace \\
\hline $\begin{array}{l}\text { Técnico Avanzado en } \\
\text { Metodología para la } \\
\text { Generación de Inteligencia } \\
\text { en el Nivel Estratégico. }\end{array}$ & NP & $\begin{array}{l}\text { Campus Internacional para la } \\
\text { Seguridad y Defensa (CISDE) }\end{array}$ & Oct & $\begin{array}{l}\frac{\mathrm{http} / / / \text { cisde.es/catalogo-de- }}{\frac{\text { cursos/inteligencia-e- }}{\text { informacion/tecnico- }}} \\
\frac{\text { avanzado-en-metodologia- }}{\text { para-la-generacion-de- }} \\
\frac{\text { inteligencia-en-el-nivel- }}{\text { estrategico }}\end{array}$ \\
\hline $\begin{array}{l}\text { Técnico Avanzado en } \\
\text { Fundamentos de } \\
\text { Inteligencia y } \\
\text { Contrainteligencia. }\end{array}$ & NP & $\begin{array}{l}\text { Campus Internacional para la } \\
\text { Seguridad y Defensa (CISDE) }\end{array}$ & Mar & $\begin{array}{l}\frac{\text { http://cisde.es/catalogo-de- }}{\text { cursos/inteligencia-e- }} \\
\begin{array}{c}\text { informacion/tecnico- } \\
\text { avanzado-en-fundamentos- }\end{array} \\
\text { de-inteligencia-y- } \\
\text { contrainteligencia-2 }\end{array}$ \\
\hline $\begin{array}{l}\text { Técnico Avanzado en } \\
\text { Servicios de Inteligencia: } \\
\text { Organización e Historia. }\end{array}$ & NP & $\begin{array}{l}\text { Campus Internacional para la } \\
\text { Seguridad y Defensa (CISDE) }\end{array}$ & Dic & $\begin{array}{l}\frac{\text { http://cisde.es/catalogo-de- }}{\text { cursos/inteligencia-e- }} \\
\underline{\text { informacion/tecnico- }} \\
\frac{\text { avanzado-en-servicios-de- }}{\text { inteligencia-organizacion- }} \\
\text { e-historia }\end{array}$ \\
\hline $\begin{array}{c}\text { Historia de los servicios de } \\
\text { inteligencia. }\end{array}$ & NP & $\begin{array}{l}\text { Campus Internacional para la } \\
\text { Seguridad y Defensa (CISDE) }\end{array}$ & Mar & $\begin{array}{c}\underline{\text { http://cisde.es/catalogo-de- }} \\
\begin{array}{c}\text { cursos/especializacion- } \\
\text { profesional/historia-de-los- }\end{array} \\
\underline{\text { servicios-de-inteligencia-2 }}\end{array}$ \\
\hline $\begin{array}{l}\text { Técnicas en Obtención } \\
\text { OSINT y Análisis } \\
\text { Estratégico STRATINT. }\end{array}$ & NP & $\begin{array}{l}\text { Campus Internacional para la } \\
\text { Seguridad y Defensa (CISDE) }\end{array}$ & Nov & $\frac{\underline{\text { http://campus.cisde.es/course } / \mathrm{i}}}{\text { nfo.php?id=279 }}$ \\
\hline
\end{tabular}




\begin{tabular}{|c|c|c|c|c|}
\hline $\begin{array}{l}\text { Técnico Avanzado en } \\
\text { Obtención de Información } \\
\text { en Fuentes Abiertas } \\
\text { (OSINT). }\end{array}$ & NP & $\begin{array}{l}\text { Campus Internacional para la } \\
\text { Seguridad y Defensa (CISDE) }\end{array}$ & $\mathrm{Ab}$ & $\begin{array}{l}\frac{\text { http://cisde.es/catalogo-de- }}{\text { cursos/inteligencia-e- }} \\
\text { informacion/tecnico- } \\
\text { avanzado-en-obtencion-de- } \\
\frac{\text { informacion-en-fuentes- }}{\text { abiertas-osint }}\end{array}$ \\
\hline $\begin{array}{l}\text { Curso de Introducción al } \\
\text { Interrogatorio. }\end{array}$ & NP & $\begin{array}{l}\text { Campus Internacional para la } \\
\text { Seguridad y Defensa (CISDE) }\end{array}$ & Ene & $\begin{array}{l}\text { http://cisde.es/catalogo-de- } \\
\frac{\text { cursos/especializacion- }}{\text { profesional/curso-de- }} \\
\text { introduccion-al-interrogatorio- }\end{array}$ \\
\hline $\begin{array}{c}\text { Técnico Avanzado en } \\
\text { HUMINT: Técnicas de } \\
\text { Inteligencia Clásica, } \\
\text { Espionaje y } \\
\text { Contraespionaje. }\end{array}$ & NP & $\begin{array}{l}\text { Campus Internacional para la } \\
\text { Seguridad y Defensa (CISDE) }\end{array}$ & Sept & $\begin{array}{l}\frac{\mathrm{http}: / / \text { cisde.es/catalogo-de- }}{\mathrm{cursos} / \text { inteligencia-e- }} \\
\underline{\text { informacion/tecnico- }} \\
\text { avanzado-en-humint-tecnicas- } \\
\begin{array}{c}\text { de-inteligencia-clasica- } \\
\text { espionaje-y-contraespionaje }\end{array}\end{array}$ \\
\hline $\begin{array}{l}\text { HUMINT: Comunicación } \\
\text { No-Verbal en Operaciones } \\
\text { de Inteligencia y } \\
\text { Contrainteligencia. }\end{array}$ & NP & $\begin{array}{l}\text { Campus Internacional para la } \\
\text { Seguridad y Defensa (CISDE) }\end{array}$ & Mar & $\frac{\underline{\text { http: } / / \text { campus.cisde.es } / \text { cour }}}{\underline{\text { se/info.php?id=405 }}}$ \\
\hline $\begin{array}{l}\text { Técnico Avanzado en } \\
\text { Inteligencia Sanitaria } \\
\text { (MEDINT). }\end{array}$ & NP & $\begin{array}{l}\text { Campus Internacional para la } \\
\text { Seguridad y Defensa (CISDE) }\end{array}$ & Mar & $\begin{array}{l}\frac{\text { http://cisde.es/catalogo-de- }}{\text { cursos/inteligencia-e- }} \\
\frac{\text { informacion/tecnico- }}{\text { avanzado-en-inteligencia- }} \\
\text { sanitaria-medint }\end{array}$ \\
\hline $\begin{array}{l}\text { Curso Avanzado de Ciber- } \\
\text { seguridad en Inteligencia: } \\
\text { Obtención, Custodia y } \\
\text { Difusión de Información. }\end{array}$ & $\mathrm{NP}$ & $\begin{array}{l}\text { Campus Internacional para la } \\
\text { Seguridad y Defensa (CISDE) }\end{array}$ & May & $\begin{array}{l}\frac{\mathrm{http} / / \text { cisde.es/catalogo-de- }}{\text { cursos/especializacion- }} \\
\text { profesional/ciberseguridad-en- } \\
\frac{\text { inteligencia-obtencion- }}{\text { custodia-y-difusion-de- }} \\
\underline{\text { informacion-2 }}\end{array}$ \\
\hline $\begin{array}{l}\text { Vigilancia Tecnología e } \\
\text { Inteligencia Competitiva. }\end{array}$ & $\mathrm{NP}$ & IALE Tecnológica & - & $\begin{array}{l}\frac{\text { http://www.ialetecnologia.c }}{\text { om/wp- }} \\
\underline{\text { content/uploads/VTIC.pdf }}\end{array}$ \\
\hline $\begin{array}{l}\text { Herramientas de Análisis } \\
\text { para la Vigilancia } \\
\text { Tecnológica e Inteligencia } \\
\text { Competitiva. }\end{array}$ & $\mathrm{NP}$ & IALE Tecnológica & - & $\begin{array}{l}\frac{\text { http://www.ialetecnologia.c }}{\text { om/wp- }} \\
\frac{\text { content/uploads/HAVTIC.p }}{\underline{\mathrm{df}}}\end{array}$ \\
\hline $\begin{array}{l}\text { Herramientas de Internet } \\
\text { para la Vigilancia } \\
\text { Tecnológica e Inteligencia } \\
\text { Competitiva. }\end{array}$ & $\mathrm{NP}$ & IALE Tecnológica & - & $\begin{array}{l}\frac{\text { http://www.ialetecnologia.c }}{\frac{\text { om/wp- }}{\text { content/uploads/HIVTIC.pd }}} \\
\underline{\mathrm{f}}\end{array}$ \\
\hline $\begin{array}{l}\text { Previsión Tecnológica y } \\
\text { Prospectiva. }\end{array}$ & $\mathrm{NP}$ & IALE Tecnológica & - & $\begin{array}{l}\frac{\text { http://www.ialetecnologia.c }}{\text { om/wp- }} \\
\underline{\text { content/uploads/PTP.pdf }}\end{array}$ \\
\hline $\begin{array}{l}\text { Metodología para la } \\
\text { Vigilancia Tecnológica e } \\
\text { Inteligencia Competitiva. }\end{array}$ & $\mathrm{P}$ & $\begin{array}{c}\text { Centro de Vigilancia Normas } \\
\text { y Patentes (CDE) }\end{array}$ & Nov & $\begin{array}{l}\frac{\text { http://www.cde.es/archivos/ }}{\text { antes/Cursos-CDE- }} \\
\frac{\text { Inteligencia-Competitiva- }}{\text { Noviembre-2014.pdf }}\end{array}$ \\
\hline
\end{tabular}




\begin{tabular}{|c|c|c|c|c|}
\hline $\begin{array}{l}\text { Sindicación Avanzada para } \\
\text { la vigilancia tecnológica e } \\
\text { Inteligencia competitiva }\end{array}$ & $P$ & $\begin{array}{c}\text { Centro de Vigilancia Normas } \\
\text { y Patentes (CDE) }\end{array}$ & Nov & $\begin{array}{l}\frac{\text { http://www.cde.es/archivos/ }}{\text { antes/Cursos-CDE- }} \\
\frac{\text { Inteligencia-Competitiva- }}{\text { Noviembre-2014.pdf }}\end{array}$ \\
\hline $\begin{array}{l}\text { Curso Superior de } \\
\text { Inteligencia. }\end{array}$ & $\mathrm{P}+\mathrm{NP}$ & $\begin{array}{l}\text { Centro Superior de Estudios } \\
\text { de la Defensa Nacional } \\
\text { (CESEDEN) }\end{array}$ & Oct- jun & $\frac{\frac{\text { http://www.defensa.gob.es/ }}{\text { ceseden/cursos/curSuperInt }}}{\lfloor}$ \\
\hline $\begin{array}{l}\text { Experto en Análisis de } \\
\text { Inteligencia }\end{array}$ & $\mathrm{P}+\mathrm{NP}$ & $\begin{array}{c}\text { Universidad Autónoma de } \\
\text { Madrid / ICFS }\end{array}$ & $\begin{array}{l}\text { Septiembre- } \\
\text { Septiembre(+ } \\
\text { 1) }\end{array}$ & $\begin{array}{l}\frac{\text { http://www.uam.es/ss/Satellite }}{\text { /es/1242654675830/12426701 }} \\
\text { 22916/estudiopropio/estudioPr } \\
\text { opio/Experto_en_Analisis_de } \\
\text { Inteligencia.htm }\end{array}$ \\
\hline $\begin{array}{l}\text { Ciclo de formación: } \\
\text { Habilidades para la } \\
\text { Inteligencia }\end{array}$ & $P$ & Universidad Autónoma/ ICFS & - & $\frac{\underline{\text { https://eei.icfs.es/formacion/ci }}}{\text { clo-de-formacion-habilidades- }}$ \\
\hline $\begin{array}{l}\text { Ciclo de formación en } \\
\text { Inteligencia Económica }\end{array}$ & $P$ & Universidad Autónoma/ ICFS & - & $\frac{\frac{\text { https://eei.icfs.es/formacion/ci }}{\text { clo-de-formacion-habilidades- }}}{\text { para-la-inteligencia }}$ \\
\hline $\begin{array}{c}\text { Certificado de } \\
\text { Especialización Profesional } \\
\text { en Análisis de Inteligencia } \\
\text { Financiera }\end{array}$ & NP & $\begin{array}{l}\text { Universidad de Valencia / } \\
\text { IUICCP }\end{array}$ & Marzo - Junio & $\begin{array}{l}\frac{\underline{\text { http://postgrado.adeit- }}}{\text { uv.es/es/cursos/juridica_y s }} \\
\frac{\text { 2/14226020/datos_generale }}{\text { ocial- }} \\
\underline{\text { s.htm?pdf }=14226020}\end{array}$ \\
\hline $\begin{array}{c}\text { Curso Especializado en } \\
\text { Inteligencia Competitiva y } \\
\text { Marketing }\end{array}$ & $\mathrm{P}$ & ESIC & $\begin{array}{c}\text { Febrero - } \\
\text { Mayo }\end{array}$ & $\begin{array}{l}\frac{\text { http://www.esic.edu/executi }}{\text { ve programa corta duraci }} \\
\frac{\text { on/3433/e/curso- }}{\text { especializado-de- }} \\
\frac{\text { inteligencia-competitiva- }}{\text { marketing }}\end{array}$ \\
\hline $\begin{array}{l}\text { Curso Operativo de } \\
\text { Seguridad e Inteligencia }\end{array}$ & $P$ & Universidad de Alicante & Marzo & $\begin{array}{l}\frac{\text { http://m.deje.ua.es/es/cienci }}{\text { a-politica- }} \\
\frac{\text { administracion/curso- }}{\text { operativo-de-seguridad-e- }} \\
\frac{\text { inteligencia-segunda- }}{\text { edicion.html }}\end{array}$ \\
\hline $\begin{array}{c}\text { Programa Ejecutivo de } \\
\text { Inteligencia de Negocio \& } \\
\text { Big Data }\end{array}$ & $P$ & $\begin{array}{c}\text { Escuela de Organización } \\
\text { Industrial }\end{array}$ & $\begin{array}{l}\text { Noviembre - } \\
\text { Junio }\end{array}$ & $\begin{array}{l}\frac{\mathrm{http}: / / \text { www.eoi.es/portal/gu }}{\text { est/curso/58/programa- }} \\
\frac{\text { ejecutivo-de-inteligencia- }}{\text { de-negocio-big-data- }} \\
\text { madrid?EOI tipoPagina }=1\end{array}$ \\
\hline
\end{tabular}




\begin{tabular}{|c|c|c|c|c|}
\hline $\begin{array}{c}\text { Curso Superior } \\
\text { Inteligencia, Intervención y } \\
\text { Protección }\end{array}$ & $\mathrm{P}$ & $\begin{array}{c}\text { Guardian Spain Homeland } \\
\text { Security }\end{array}$ & Noviembre & $\begin{array}{l}\frac{\text { http://www.guardianspain.c }}{\text { om/curso-superior- }} \\
\frac{\text { inteligencia-intervencion-y- }}{\text { proteccion/ }}\end{array}$ \\
\hline $\begin{array}{c}\text { Curso en Inteligencia } \\
\text { Criminal }\end{array}$ & $\mathrm{P}$ & $\begin{array}{c}\text { Universitat Autónoma de } \\
\text { Barcelona }\end{array}$ & Enero-Mayo & $\begin{array}{c}\frac{\text { http://www.uab.cat/web/po }}{\frac{\text { stgrado/curso-en- }}{\text { inteligencia- }}} \\
\frac{\text { criminal/informacion- }}{\text { general- }} \\
\frac{1206597475768 . h t m l / \text { para }}{\text { m1-3035 es/param2-2008/ }}\end{array}$ \\
\hline $\begin{array}{c}\text { Curso Monográfico de } \\
\text { Inteligencia Empresarial } \\
\text { para la Toma de Decisiones } \\
\text { Efectivas }\end{array}$ & $\mathrm{P}$ & $\begin{array}{l}\text { Centro de Estudios } \\
\text { Financieros }\end{array}$ & $\begin{array}{l}\text { Octubre - } \\
\text { Marzo }\end{array}$ & $\begin{array}{c}\frac{\text { http://www.cef.es/Cursos/c }}{\text { urso-monografico- }} \\
\text { inteligencia-empresarial- } \\
\text { para-toma-de-decisiones- } \\
\frac{\text { efectivas-business- }}{\text { intelligence.html }}\end{array}$ \\
\hline $\begin{array}{c}\text { Curso de Inteligencia } \\
\text { Aplicada }\end{array}$ & $\mathrm{P}$ & $\begin{array}{l}\text { Universidad Nacional de } \\
\text { Educación a Distancia } \\
\text { (UNED) }\end{array}$ & Diciembre & $\frac{\text { http://extension.uned.es/acti }}{\underline{\text { vidad/idactividad/8251 }}}$ \\
\hline $\begin{array}{c}\text { Curso de Inteligencia } \\
\text { Económica y Competitiva }\end{array}$ & $\mathrm{P}$ & Grupo IberCaja & $\begin{array}{l}\text { Noviembre- } \\
\text { Diciembre }\end{array}$ & $\begin{array}{l}\frac{\text { https://obrasocial.ibercaja.e }}{\text { s/iniciativa- }} \\
\frac{\text { emplea/zaragoza/curso-de- }}{\text { inteligencia-economica-y- }} \\
\text { competitiva }\end{array}$ \\
\hline $\begin{array}{l}\text { Inteligencia en la Guerra } \\
\text { Civil Norteamericana }\end{array}$ & NP & Instituto de Seguridad Global & - & $\begin{array}{l}\text { http://catalogo.institutodese } \\
\text { guridadglobal.com/catalogo } \\
\text { /moocs/inteligencia-en-la- } \\
\text { guerra-civil- } \\
\text { norteamericana/ }\end{array}$ \\
\hline $\begin{array}{l}\text { Taller de Inteligencia } \\
\text { Competitiva }\end{array}$ & $\mathrm{P}$ & Help 4 Decision Makers & - & $\frac{\underline{\mathrm{http}: / / \mathrm{h} 4 \mathrm{dm} \cdot \mathrm{com} / \text { formacion/cu }}}{\frac{\text { rso-de-inteligencia- }}{\underline{\text { competitiva }}}}$ \\
\hline $\begin{array}{l}\text { Curso de herramientas de } \\
\text { análisis de inteligencia }\end{array}$ & $\mathrm{P}$ & Help 4 Decision Makers & - & $\frac{\text { http://h4dm.com/formacion }}{\text { /curso-de-herramientas-de- }}$ \\
\hline $\begin{array}{l}\text { Vigilancia Tecnológica e } \\
\text { Inteligencia Competitiva }\end{array}$ & $\mathrm{P}$ & AENOR & Mayo & $\begin{array}{l}\frac{\text { http://www.aenor.es/aenor/f }}{\text { ormacion/cursos/fichacurso }} \\
\frac{. \text { asp?curso=I- }}{\overline{04 \& \text { anyo=2015\&modal ori }}} \\
\text { gen=PR\#.VM1QNC5CC1s }\end{array}$ \\
\hline $\begin{array}{l}\text { Curso Intensivo "Técnicas } \\
\text { Operativas de Inteligencia } \\
\text { Corporativa" }\end{array}$ & $\mathrm{P}$ & Globalchase & Mayo & https://intel.global/formacion \\
\hline $\begin{array}{c}\text { Curso Internacional de } \\
\text { Inteligencia y Prospectiva }\end{array}$ & NP & UNED/ Globalchase & Oct-Nov & $\frac{\text { https://extension.uned.es/activ }}{\text { idad/idactividad/4086 }}$ \\
\hline
\end{tabular}




\begin{tabular}{|c|c|c|c|c|}
\hline $\begin{array}{l}\text { Técnico Avanzado de } \\
\text { Análisis de información en } \\
\text { inteligencia y seguridad }\end{array}$ & NP & $\begin{array}{l}\text { Campus Internacional para la } \\
\text { Seguridad y Defensa (CISDE) }\end{array}$ & Febrero & $\begin{array}{l}\frac{\underline{\text { http://cisde.es/catalogo-de- }}}{\underline{\text { cursos/inteligencia-e- }}} \\
\text { informacion/tecnico- } \\
\text { avanzado-en-metodos-de- } \\
\text { analisis-de-informacion-en- } \\
\text { inteligencia-y-seguridad }\end{array}$ \\
\hline $\begin{array}{c}\text { Curso diplomacia en } \\
\text { Inteligencia Competitiva y } \\
\text { Marketing }\end{array}$ & $\mathrm{NP}$ & ESIC & Febrero-mayo & $\begin{array}{l}\frac{\mathrm{http}: / / \text { www.esic.edu/executi }}{\text { ve programa corta duraci }} \\
\frac{\text { on/3433/e/curso- }}{\text { especializado-de- }} \\
\frac{\text { inteligencia-competitiva- }}{\text { marketing }}\end{array}$ \\
\hline $\begin{array}{c}\text { Curso Operativo de } \\
\text { Seguridad e Inteligencia }\end{array}$ & $P$ & Universidad de Alicante & Marzo & $\begin{array}{l}\frac{\text { http://m.deje.ua.es/es/cienci }}{\text { a-politica- }} \\
\frac{\text { administracion/curso- }}{\text { operativo-de-seguridad-e- }} \\
\frac{\text { inteligencia-segunda- }}{\text { edicion.html }}\end{array}$ \\
\hline $\begin{array}{c}\text { Curso en Inteligencia } \\
\text { Competitiva y Vigilancia } \\
\text { Tecnológica }\end{array}$ & NP & Universitat Pompeu Fabra & $\begin{array}{l}\text { Noviembre- } \\
\text { Febrero }\end{array}$ & $\begin{array}{l}\frac{\text { http://www.barcelonaschoo }}{\text { lofmanagement.upf.edu/cur }} \\
\frac{\text { so-en-inteligencia- }}{\text { competitiva-y-vigilancia- }} \\
\text { tecnologica/presentacion }\end{array}$ \\
\hline $\begin{array}{l}\text { Especialista Universitario } \\
\text { en Servicios de Inteligencia }\end{array}$ & $\mathrm{P}+\mathrm{NP}$ & $\begin{array}{l}\text { UNED / Instituto } \\
\text { Universitario General } \\
\text { Gutiérrez Mellado }\end{array}$ & $\begin{array}{l}\text { Noviembre - } \\
\text { Septiembre }\end{array}$ & $\begin{array}{l}\frac{\text { http://iugm.es/docencia/for }}{\text { macion- }} \\
\frac{\text { continua/especialista- }}{\text { universitario/servicios-de- }} \\
\text { inteligencia/ }\end{array}$ \\
\hline $\begin{array}{l}\text { Servicios de Inteligencia y } \\
\text { Seguridad Internacional }\end{array}$ & $\mathrm{P}$ & Universidad de Alicante & Marzo & $\begin{array}{l}\frac{\text { http://m.deje.ua.es/es/cienci }}{\text { a-politica- }} \\
\frac{\text { administracion/curso- }}{\text { operativo-de-seguridad-e- }} \\
\frac{\text { inteligencia-segunda- }}{\text { edicion.html }}\end{array}$ \\
\hline $\begin{array}{c}\text { Curso Superior de } \\
\text { Inteligencia Económica y } \\
\text { Empresarial }\end{array}$ & NP & $\begin{array}{l}\text { Universidad Francisco de } \\
\text { Vitoria / Grupo ATENEA }\end{array}$ & $\begin{array}{c}\text { Abril - } \\
\text { Noviembre }\end{array}$ & $\begin{array}{l}\frac{\text { http://www.grupoateneasd. }}{\text { es/wp- }} \\
\frac{\text { content/uploads/2014/12/C }}{\text { URSO-INTELG-ECON-Y- }} \\
\frac{\text { EMPRESARIAL-01-DIC- }}{\text { 2014.pdf }}\end{array}$ \\
\hline $\begin{array}{c}\text { Curso Intensivo de } \\
\text { “Inteligencia Operativa y } \\
\text { Táctica” }\end{array}$ & $\mathrm{P}$ & FESEI & Septiembre & $\frac{\frac{\mathrm{http}: / / \text { www.cesei.es/upload }}{\text { s/1/3/6/1/13611746/curso_i }}}{\frac{\text { nteligencia_operativa_y_tct }}{\text { ica.pdf }}}$ \\
\hline $\begin{array}{l}\text { Curso Especializado en } \\
\text { Técnicas de Inteligencia y } \\
\text { Prospectiva Estratégica }\end{array}$ & $\mathrm{P}$ & FESEI & N/A & $\begin{array}{l}\frac{\text { http://www.cesei.es/curso- }}{\text { especializado-en- }} \\
\frac{\text { teacutecnicas-de- }}{\text { inteligencia-y-prospectiva- }} \\
\text { estrateacutegica.html }\end{array}$ \\
\hline $\begin{array}{l}\text { Curso de Especialista en } \\
\text { Inteligencia Turística }\end{array}$ & NP & $\begin{array}{l}\text { Escuela Internacional de } \\
\text { Inteligencia Turística }\end{array}$ & Julio & $\frac{\text { http://escuela.inteligenciatu }}{\text { ristica.com/index.php }}$ \\
\hline
\end{tabular}




\begin{tabular}{|c|c|c|c|c|}
\hline $\begin{array}{l}\text { Análisis de la Seguridad e } \\
\text { Inteligencia }\end{array}$ & P-NP & Universidad de Barcelona & Nov-Sept & $\begin{array}{l}\frac{\text { http://www.ub.edu/web/ub/ }}{\text { es/estudis/oferta formativa/ }} \\
\frac{\text { masters propis/fitxa/A/201 }}{311856 / \text { index.htm } 1}\end{array}$ \\
\hline $\begin{array}{c}\text { Curso de Inteligencia } \\
\text { Operativa }\end{array}$ & $\mathrm{P}$ & ADI Spain Security Training & - & $\frac{\text { http://www.adispain.com/in }}{\text { teligencia/curso-de- }}$ \\
\hline $\begin{array}{l}\text { Curso Superior de } \\
\text { Inteligencia Competitiva }\end{array}$ & $\mathrm{P}$ & Centro Tecnológico IK4-Ideko & Nov & $\begin{array}{l}\frac{\mathrm{http}: / / \text { www.intelsuite.com/e }}{\mathrm{s} / \mathrm{blog} / \text { intelsuite-te-invita-a- }} \\
\text { participar-en-el-curso- } \\
\frac{\text { superior-de-inteligencia- }}{\text { competitiva }}\end{array}$ \\
\hline $\begin{array}{l}\text { I Curso de Inteligencia } \\
\text { Económica }\end{array}$ & $\mathrm{P}$ & $\begin{array}{l}\text { Instituto de Probática e } \\
\text { Investigación Criminal }\end{array}$ & Feb & $\begin{array}{l}\underline{\mathrm{http}: / / \text { www.icriminal.org/co }} \\
\underline{\text { urses/curso-de-inteligencia- }} \\
\underline{\text { economica/ }}\end{array}$ \\
\hline $\begin{array}{l}\text { Análisis de la Seguridad e } \\
\text { Inteligencia (Experto) }\end{array}$ & $\mathrm{P}+\mathrm{NP}$ & Universidad de Barcelona & Nov & $\begin{array}{l}\frac{\text { http://www.ub.edu/web/ub/ }}{\text { es/estudis/oferta formativa/ }} \\
\frac{\text { masters_propis/fitxa/A/201 }}{\underline{311856 / \text { index.html }}}\end{array}$ \\
\hline $\begin{array}{c}\text { Inteligencia y Seguridad. } \\
\text { Análisis del fenómeno } \\
\text { Yihadista }\end{array}$ & $\mathrm{P}$ & Universidad de La Rioja & Jun & 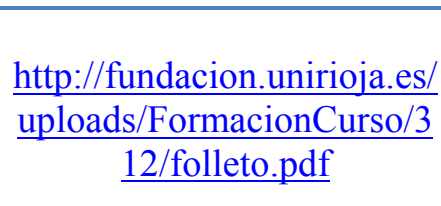 \\
\hline $\begin{array}{c}\text { I Curso Superior de } \\
\text { Terrorismo, Inteligencia y } \\
\text { Contramedidas }\end{array}$ & $\mathrm{P}+\mathrm{NP}$ & $\begin{array}{c}\text { Universidad Rey Juan Carlos, } \\
\text { Escuela Europea de Ciencias } \\
\text { de la Seguridad }\end{array}$ & Oct-Jul & $\begin{array}{l}\frac{\text { http://www.eecs.es/cursos- }}{\text { con-la-universidad-rey- }} \\
\text { juan-carlos/curso-superior- } \\
\frac{\text { terrorismo-inteligencia- }}{\text { contramedidas/ }}\end{array}$ \\
\hline $\begin{array}{l}\text { Diseño de Sistemas de } \\
\text { Vigilancia Tecnológica e } \\
\text { Inteligencia Competitiva. }\end{array}$ & NP & IALE Tecnológica & - & $\begin{array}{l}\underline{\text { http://www.ialetecnologia.co }} \\
\underline{\mathrm{m} / \mathrm{wp}-} \\
\text { content/uploads/DSVTIC.pdf }\end{array}$ \\
\hline $\begin{array}{c}\text { Curso Especializado } \\
\text { Inteligencia Económica y } \\
\text { Diplomacia Económica }\end{array}$ & $\mathrm{P}$ & $\begin{array}{l}\text { Instituto Atlántico de } \\
\text { Gobierno }\end{array}$ & Jun & $\begin{array}{l}\frac{\mathrm{http}: / / \text { www.institutoatlantic }}{\text { odegobierno.org/inteligenci }} \\
\frac{\text { a-economica-y-diplomacia- }}{\text { economica/ }}\end{array}$ \\
\hline
\end{tabular}

